APS/123-QED

\title{
Multinucleon transfer reactions in closed-shell nuclei
}

\author{
S. Szilner ${ }^{1,4}$, C. A. Ur ${ }^{2,5}$, L. Corradi ${ }^{1}$, N. Mărginean ${ }^{1}$, G. Pollarolo ${ }^{3}$, A. M. Stefanini ${ }^{1}$, \\ S. Beghini ${ }^{2}$, B. R. Behera ${ }^{1}$, E. Fioretto ${ }^{1}$, A. Gadea ${ }^{1}$, B. Guiot ${ }^{1}$, A. Latina ${ }^{1}$, P. Mason ${ }^{2}$, \\ G. Montagnoli' ${ }^{2}$ F. Scarlassara ${ }^{2}$, M. Trotta ${ }^{7}$, G. de Angelis ${ }^{1}$, F. Della Vedova ${ }^{2}$, E. Farnea ${ }^{2}$, \\ F. $\operatorname{Haas}^{6}$, S. Lenzi ${ }^{2}$, S. Lunardi ${ }^{2}$, R. Mărginean ${ }^{2}$, R. Menegazzo ${ }^{2}$, D. R. Napoli ${ }^{1}$, M. Nespolo ${ }^{2}$, \\ I. V. Pokrovsky ${ }^{1}$, F. Recchia ${ }^{2}$, M. Romoli ${ }^{7}$, M.-D. Salsac ${ }^{6}$, N. Soić ${ }^{4}$, and J. J. Valiente-Dobón ${ }^{1}$ \\ ${ }^{1}$ Istituto Nazionale di Fisica Nucleare, Laboratori Nazionali di Legnaro, I-35020 Legnaro, Italy \\ ${ }^{2}$ Dipartimento di Fisica, Università di Padova, and Istituto Nazionale di Fisica Nucleare, I-35131 Padova, Italy \\ 3 Dipartimento di Fisica Teorica, Università di Torino, \\ and Istituto Nazionale di Fisica Nucleare, 10125 Torino, Italy \\ 4 Ruđer Bošković Institute, HR-10001 Zagreb, Croatia \\ ${ }^{5}$ Horia Hulubei National Institute of Physics and Nuclear Engineering, 077125, Bucharest-Magurele, Romania \\ ${ }^{6}$ Laboratoire Pluridisciplinaire Hubert Curien, CNRS-IN2P3/ULP, F-67037 Strasbourg, France and \\ 7 Istituto Nazionale di Fisica Nucleare, Sezione di Napoli, I-80126 Napoli, Italy
}

(Dated: November 13, 2018)

\begin{abstract}
Multinucleon transfer reactions in ${ }^{40} \mathrm{Ca}+{ }^{96} \mathrm{Zr}$ and ${ }^{90} \mathrm{Zr}+{ }^{208} \mathrm{~Pb}$ have been measured at energies close to the Coulomb barrier in a high resolution $\gamma$-particle coincidence experiment. The large solid angle magnetic spectrometer PRISMA coupled to the CLARA $\gamma$-array has been employed. Trajectory reconstruction has been applied for the complete identification of transfer products. Mass and charge yields, total kinetic energy losses, $\gamma$ transitions of the binary reaction partners, and comparison of data with semiclassical calculations are reported. Specific transitions in ${ }^{95} \mathrm{Zr}$ populated in one particle transfer channels are discussed in terms of particle-phonon couplings. The $\gamma$ decays from states in ${ }^{42} \mathrm{Ca}$ in the excitation energy region expected from pairing vibrations are also observed.
\end{abstract}

PACS numbers: 25.70.Hi; 29.30.Aj; 24.10.-i; 23.20.Lv

\section{INTRODUCTION}

Our understanding of the structure of nuclei greatly benefited from reactions induced by a very large variety of probes. With photons, electrons, mesons and nucleons we learned about the properties of nuclei in the vicinity of their ground states. These results were essential for the formulation of nuclear models in terms of elementary modes like collective surface vibrations and single particle degrees of freedom. Transfer reactions induced by light ions, deuteron, tritium and alphas played an essential role for our knowledge of particle-particle correlations and led to the recognition of new elementary modes like pairvibration and pair-rotation [1].

The acceleration of heavy ions offered the possibility to bring together two complex systems. In the collision process they might exchange several quanta, of energy and angular momentum and of mass and charge, or they could fuse giving rise to compound system with very large excitation energy and intrinsic angular momentum [2]. By using heavy-ion fusion reactions the study of the nuclear structure has been extended in regions of very high angular momentum and large excitation energies. This field of research turned out to be very prolific and for the last thirty years dominated the nuclear structure studies. However, other aspects of the nuclear behavior [3] remain largely unexplored. For instance the existence of the two octupole phonons in ${ }^{208} \mathrm{~Pb}$ is still debated [4, 5, 6], and only recently the particle-vibration coupling scheme received new attention from the observation of large sur- vival probabilities of the octupole vibration in all neighboring nuclei populated in deep-inelastic or multi-nucleon transfer reactions with lead [7].

The identification of elementary modes of excitation in nuclei turned out to be important also for the development of models for the reaction. While it is natural to use the semi-classical approximation for the description of the relative motion, the coherent excitation of the elementary modes (multi-phonons) allows the use of the same semi-classical approximation also for the treatment of the intrinsic degrees of freedom and thus to develop models that are able to treat on the same footing phonons and single particle degrees of freedom [8, 9].

Heavy-ion reactions offer, in principle, an ideal tool for the study of the residual interaction in nuclei, in particular the components responsible for the couplings between the phonon degrees of freedom and the single particle (particle-vibration coupling), and via multi-nucleon transfer reactions the component responsible for particle correlations like the pairing interaction. However, the analysis and subsequent interpretation of these reactions turned out to be quite complex being the information about correlations buried in the inclusive character of the extracted cross sections [10, 11]. With the ability to measure individual transitions [12], a deeper insight into particle correlations can be achieved. The new generation of large solid angle spectrometers [13, 14] coupled with $\gamma$ arrays [15, 16] gives the possibility to look at individual transitions, their population pattern and decay modes via particle- $\gamma$ coincidences. The need to reach good res- 
olution in all mass regions and to measure low cross sections for massive transfer channels, lead to the construction of PRISMA+CLARA. In the magnetic spectrometer PRISMA [13] reaction products are identified via an event-by-event reconstruction of the ion trajectory inside the magnetic elements. Coincident $\gamma$ rays are detected with CLARA 15], where its high granularity allows to perform precise Doppler correction of transitions tagged with the spectrometer.

In this manuscript the multinucleon transfer reactions in ${ }^{40} \mathrm{Ca}+{ }^{96} \mathrm{Zr}$ and ${ }^{90} \mathrm{Zr}+{ }^{208} \mathrm{~Pb}$, studied at energies close to the Coulomb barrier with the PRISMA+CLARA setup are presented. Both projectile and target are well known closed-shell nuclei and therefore optimum candidates for having clean experimental and theoretical conditions.

\section{THE EXPERIMENTAL SET-UP}

Beams of ${ }^{40} \mathrm{Ca}$ and ${ }^{90} \mathrm{Zr}$ have been accelerated on ${ }^{96} \mathrm{Zr}$ and ${ }^{208} \mathrm{~Pb}$ targets, respectively, by the Tandem and ALPI booster of LNL at bombarding energies of $152 \mathrm{MeV}$ and $560 \mathrm{MeV}$ [17], respectively. The beams had an average intensity of $\simeq 3 \mathrm{pnA}$. The ${ }^{96} \mathrm{Zr}$ (in form of oxide) and ${ }^{208} \mathrm{~Pb}$ targets had a thickness of 150 and $290 \mu \mathrm{g} / \mathrm{cm}^{2}$, respectively. Both targets had an isotopic enrichment of $99.9 \%$ and consisted of a strip of $1 \mathrm{~mm}$ on a $20 \mu \mathrm{g} / \mathrm{cm}^{2}$ C-layer.

FIG. 1: Top: Example of two-dimensional $\Delta E-E$ matrix corresponding to one section of the $\mathrm{IC}$, obtained in the reaction ${ }^{90} \mathrm{Zr}+{ }^{208} \mathrm{~Pb}$. The most intense band corresponds to $\mathrm{Z}=40$. The diagonal line is a trivial effect of detector thresholds. The matrix has been constructed by requiring the coincidence with at least one $\gamma$-ray in CLARA, so the contribution of elastic scattering is suppressed. Bottom: Two-dimensional Range vs total energy matrix obtained in the reaction ${ }^{40} \mathrm{Ca}+{ }^{96} \mathrm{Zr}$, where all sections of the IC have been included. The most intense band corresponds to $\mathrm{Z}=20$. The two bands located at the bottom of the matrix correspond to ${ }^{12} \mathrm{C}$ and ${ }^{16} \mathrm{O}$ contaminants present in the target.

Projectile-like products have been selected with the magnetic spectrometer PRISMA placed at $\theta_{\mathrm{lab}}=68^{\circ}$ for the ${ }^{40} \mathrm{Ca}+{ }^{96} \mathrm{Zr}$ and $\theta_{\text {lab }}=61^{\circ}$ for the ${ }^{90} \mathrm{Zr}+{ }^{208} \mathrm{~Pb}$ reactions. PRISMA consists of a magnetic quadrupole singlet, placed at $50 \mathrm{~cm}$ from the target, and a magnetic dipole $\left(60^{\circ}\right.$ bending angle and $1.2 \mathrm{~m}$ curvature radius). Its main characteristics are the large solid angle of $\simeq 80$ msr (corresponding to $\pm 6^{\circ}$ in $\theta$ and $\pm 11^{\circ}$ in $\phi$ ), a momentum acceptance $\Delta p / p= \pm 10 \%$ and a dispersion of $\simeq$ $4 \mathrm{~cm}$ per percent in momentum. A two-dimensional position sensitive micro-channel plate (MCP) detector [18] is placed at the entrance of the spectrometer providing a start signal for time-of-flight measurement with subnanosecond resolution and $X_{\mathrm{i}}, Y_{\mathrm{i}}$ signals with $1 \mathrm{~mm}$ res- olutions. Ions pass through the optical elements of the spectrometer and after a path of $\simeq 6.5 \mathrm{~m}$, enter a focal plane detector [19]. This is made of a parallel plate of multiwire-type (MWPPAC) divided into ten sections, providing timing and $X_{\mathrm{f}}, Y_{\mathrm{f}}$ position signals derived via a delay line method, with resolutions similar to the MCP ones. Behind the MWPPAC an array of a transverse field multiparametric ionization chambers (IC) is placed, providing nuclear charge $(\Delta E)$ and total energy $(E)$ (with a $\sim 2-3 \%$ resolution). The IC is divided into ten transversal sections (like the MWPPAC) with four $\Delta E$ subsections each. Combining the $\Delta E$ subsections and adjusting properly the gas pressure, one can stop inside the IC ions that differ by more than $20 \%$ in kinetic energy, and optimize the $Z$ and total energy resolutions.

The $\gamma$-ray array CLARA [15] consists of 24 HPGe clover-type detectors placed to form a $2 \pi$ configuration close to the target position and opposite to PRISMA. Each clover detector is composed of four crystals mounted in a single cryostat and surrounded by an anti-Compton shield, ensuring a peak-to-total ratio of $\simeq 50 \%$. The total photopeak efficiency of CLARA is of the order of $3 \%$ for $E_{\gamma}=1.33 \mathrm{MeV}$. Typical $\gamma$-ray energy resolutions obtained after Doppler correction are $0.6 \%$ to $0.9 \%$ over the whole velocity distribution of the projectile-like products detected in PRISMA.

\section{PRISMA DATA ANALYSIS}

As it is well known, a conventional magnetic spectrometer provides the ratio of the momentum over the atomic charge state. To obtain the mass, an additional parameter is needed, and a commonly used one is the timeof-flight. With very large solid angle spectrometers, like PRISMA, the mass identification of the reaction products can only be obtained via an event-by-event reconstruction of the ion trajectory inside the magnetic elements. The reconstruction of the trajectory is here obtained from the measurement of entrance detector positions $X_{\mathrm{i}}, Y_{\mathrm{i}}$, focal plane positions $X_{\mathrm{f}}, Y_{\mathrm{f}}$ and time-of-flight $\tau_{\text {TOF }}$. For the reconstruction a following fast algorithm has been employed. Taking advantage of the very large longitudinal dimension of PRISMA $(6.5 \mathrm{~m})$ with respect to the transversal one $(0.2 \mathrm{~m})$ and considering that the fringing fields can be neglected because of the large dimensions of the magnetic elements, the trajectory may be assumed to be planar after the quadrupole. These assumptions, planarity of the trajectory and the weak effect of the fringing fields, have been carefully checked with a simulation [20] of the ion transport through the spectrometer by using the actual fields and by mimicking the distribution of the reaction products with a Monte Carlo method. The trajectories are uniquely determined by two parameters, the bending radius in the dipole and the ratio of the quadrupole and dipole magnetic fields. Being the magnetic fields known, the bending radius $\rho$ remains the only parameter to be searched for. 

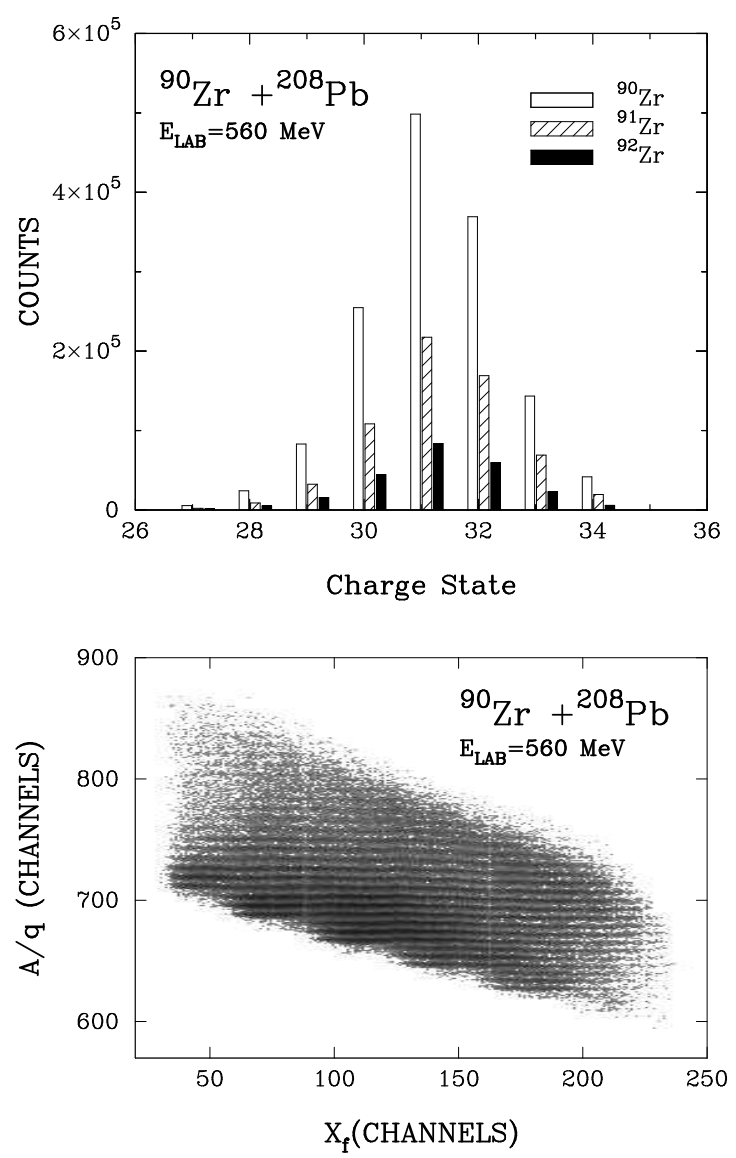

FIG. 2: Top: Atomic charge state distribution for ${ }^{90,91,92} \mathrm{Zr}$ ions selected with PRISMA in the reaction ${ }^{90} \mathrm{Zr}+{ }^{208} \mathrm{~Pb}$. Bottom: Mass over atomic charge state $A / q$ vs horizontal MWPPAC position $X_{f}$.

We briefly describe below the procedure adopted to identify the mass $A$ and the nuclear charge $Z$ of the reaction products. The identification of nuclear charge $Z$ is obtained through the measurement of energy loss $\Delta E$ in the IC, which provides also the total energy $E$. Fig. 1 (top panel) displays an example of $\Delta E-E$ matrix for the ${ }^{90} \mathrm{Zr}+{ }^{208} \mathrm{~Pb}$ system, where only one of the central sections of the IC is considered. Here a clear separation of the different $\mathrm{Z}$ is obtained. We have to keep in mind that ions reach the IC with a broad range of kinetic energies and directions, thus to get the desired $\mathrm{Z}$ resolution for all the reaction products reaching the IC, one has to properly take into account the direction followed by the different ions. One can estimate the path (Range) from the signal of each subsection and the position information of MCP and MWPPAC detectors. An example of Range vs $E$ matrix is displayed in the same figure (bottom panel) for the ${ }^{40} \mathrm{Ca}+{ }^{96} \mathrm{Zr}$ reaction. The most intense bands in Fig. 1 correspond to the $\mathrm{Z}$ of the entrance channel for both systems. As pointed out before mass identification of the reaction products requires the determination of their trajectory in the apparatus. From this trajectory we obtain the bending radius $\rho$ in the dipole and the total length $L$ up to the MWPPAC. Thus, first we obtain the quantity:

$$
\frac{B \rho \tau_{\mathrm{TOF}}}{L} \Rightarrow \frac{A}{q}
$$

that is proportional to the ratio $A / q$. By plotting this quantity as a function of the $X_{f}$ position in the MWPPAC a clear discrimination with characteristic repetitive pattern of the different $A / q$ is obtained (see bottom panel of Fig. 21). The large acceptance of the spectrometer is reflected in the fact that different atomic charge states cover several sections in the focal plane, which are all joined together in the above mentioned figure.

The actual mass is finally obtained by constructing the quantity :

$$
\frac{E \tau_{\mathrm{TOF}}}{B \rho L} \Rightarrow q
$$

that is proportional to the atomic charge state $q$. In the above equations $B$ is the strength of the dipole field and $E$ the kinetic energy of the ion.

The top panel of Fig. 2 shows, for the ${ }^{90} \mathrm{Zr}+{ }^{208} \mathrm{~Pb}$ reaction, the extracted atomic charge state distribution for different zirconium isotopes. The broad distribution, characteristic of heavy ions, does not depend on the isotope, and have a centroid that matches the one calculated according to Ref. 21]. The reconstructed mass spectra for calcium, potassium, and argon isotopes are shown in Fig. 3 for the ${ }^{40} \mathrm{Ca}+{ }^{96} \mathrm{Zr}$ reaction.

From the grazing character of these reactions we know that most of the yield in the different transfer channels is concentrated in a narrow angular range close to the grazing with a shape of the angular distribution weakly dependent on the isotope (cfr. Refs. [10, 11] and references therein for illustration). Exploiting the large angular acceptance of PRISMA one obtains a reasonable estimation of the relative production yield. The total yields for the pure neutron transfer channels are shown in Fig. 4 for the two reactions, together with the yield of the one proton stripping channel for the ${ }^{40} \mathrm{Ca}+{ }^{96} \mathrm{Zr}$ system. In the same figure the results of a semiclassical calculation [8, 22] are shown. The experimental yields have been normalized to the computed $+1 \mathrm{n}$ channel and the same normalization constant has been kept for other neutron pick-up and the proton stripping channels. The good agreement between experiment and theory gives us confidence on the correct procedure adopted for the trajectory reconstruction and of the good behavior of the device.

The dependence of the cross sections on the number of transferred neutrons is very similar to the one observed in other studied systems [10, 11, 23]. We remind that the shape of the proton stripping channels illustrates the independence between the proton and neutron transfer 


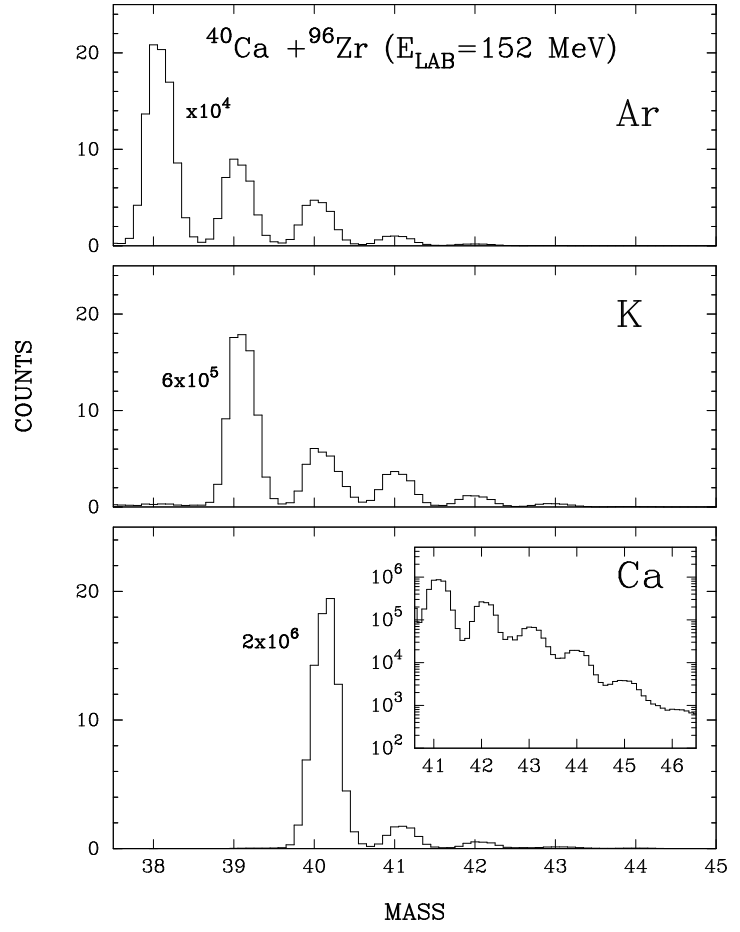

FIG. 3: Mass spectra for argon, potassium and calcium isotopes populated in the ${ }^{40} \mathrm{Ca}+{ }^{96} \mathrm{Zr}$ reaction (note the different scaling factors in each panel). The inset in the bottom panel displays the neutron pick-up channels in logarithmic scale.

degrees of freedom. The apparatus allowed the identification of neutron stripping channels which are, for stable beams, strongly suppressed by optimum $Q$-value considerations [24]. Since these channels may receive considerable contributions from evaporation processes, these effects have to be included in the theoretical model. The calculated cross sections have been obtained by using the semiclassical model GRAZING 8, 22]. This model calculates the evolution of the reaction by taking into account, besides the relative motion variables, the intrinsic degrees of freedom of projectile and target. These are the isoscalar surface modes and the single nucleon transfer channels. The multinucleon transfer channels are described via a multi-step mechanism. The relative motion of the system is calculated in a nuclear plus Coulomb field where for the nuclear part the empirical potential of Ref. [2] has been used. The excitation of the intrinsic degrees of freedom is obtained by employing the well known form factors for the collective surface vibrations and the oneparticle transfer channels [25, 26]. The model takes into account in a simple way the effect of neutron evaporation.

\section{PRISMA+CLARA DATA ANALYSIS}

The particle- $\gamma$ coincidence obtained from the coupling of CLARA with PRISMA allows to attribute to each spe-

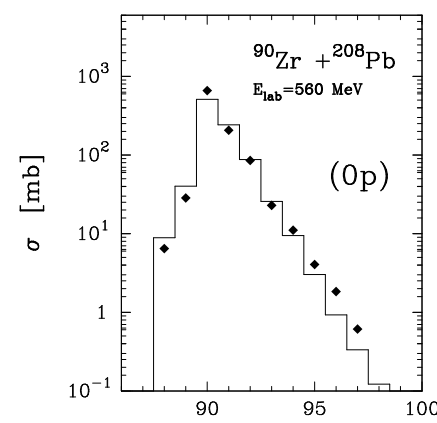

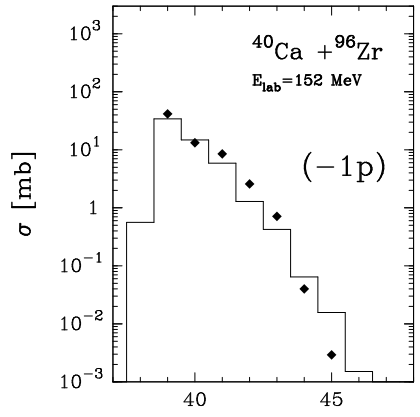

MASS NUMBER

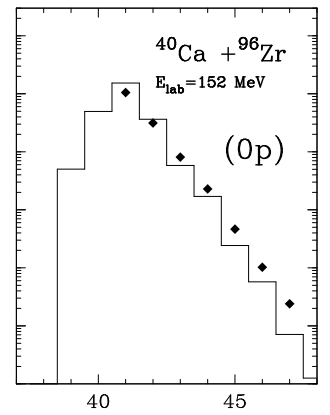

MASS NUMBER
FIG. 4: Top: Total cross sections for pure neutron pick-up channels in the ${ }^{90} \mathrm{Zr}+{ }^{208} \mathrm{~Pb}$ reaction. Bottom: Total cross sections for pure neutron pick-up (right panel) and one-proton stripping (left panel) channels in the ${ }^{40} \mathrm{Ca}+{ }^{96} \mathrm{Zr}$ reaction. The points are the experimental data and the histograms are the calculation performed with the code GRAZING.

cific reaction product its characteristic $\gamma$ rays. Since the $\gamma$ rays are emitted in-flight it is mandatory to perform Doppler correction. This is done from the knowledge of the trajectory reconstructed in PRISMA, providing the velocity vector of the emitting nuclei. The top panel of Fig. 5 shows a reconstructed velocity distribution for the ${ }^{90} \mathrm{Zr}$ ions in the ${ }^{90} \mathrm{Zr}+{ }^{208} \mathrm{~Pb}$ reaction. The middle panel depicts the corresponding Doppler corrected $\gamma$-ray spectrum, illustrating the good resolution obtained with such a procedure (the $2^{+} \rightarrow 0^{+}$transition at $2186 \mathrm{keV}$ has a FWHM of $18 \mathrm{keV}$ ). The characteristic $\gamma$ rays of the undetected heavy partner can be Doppler corrected by assuming a binary character of the reaction. In the bottom panel of Fig. 5 we display the $\gamma$-ray spectrum for ${ }^{208} \mathrm{~Pb}$, where we clearly observe the decay of the $3^{-}$and $5^{-}$states.

The present set-up offers the possibility to separate elastic from inelastic scattering. The pure elastic scattering is here determined by comparing the events with and without $\gamma$ coincidences. In the top panel of Fig. 6]are shown the total kinetic energy loss (TKEL) spectra for ${ }^{90} \mathrm{Zr}$ with and without $\gamma$-coincidence, normalized in the tail (large TKEL) region. By subtraction, we obtain the contribution of pure elastic. This subtracted spectrum is characterized by a narrow peak centered at TKEL $\simeq$ $0 \mathrm{MeV}$ with a FWHM of $2.65 \mathrm{MeV}$. Moreover, its cen- 

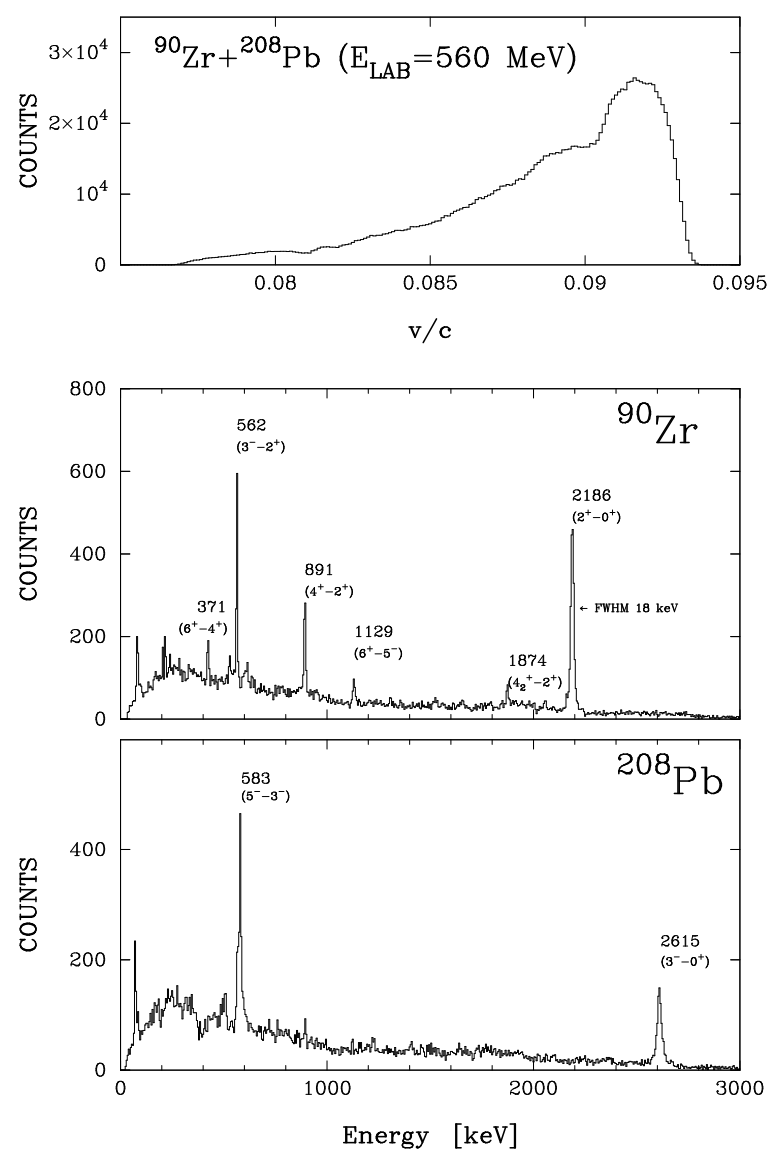

FIG. 5: Top: Reconstructed velocity distribution for the ${ }^{90} \mathrm{Zr}$ ions in the ${ }^{90} \mathrm{Zr}+{ }^{208} \mathrm{~Pb}$ reaction. Middle : Doppler corrected $\gamma$-ray spectrum for ${ }^{90} \mathrm{Zr}$. Bottom: Doppler corrected $\gamma$-ray spectrum for ${ }^{208} \mathrm{~Pb}$, obtained assuming a binary character of the reaction. The $\gamma$-ray spectra have been obtained by subtracting the wrongly Doppler corrected contribution of the binary partner. Main $\gamma$-ray transitions are marked with their energy (in keV) together with the spin and parity of the connected states.

troid is separated by $2.15 \mathrm{MeV}$ from the maximum of the TKEL spectrum in coincidence with CLARA, whose value is very close to the inelastic excitation of the first $2^{+}$ state in ${ }^{90} \mathrm{Zr}$. Such a procedure should be reliable, provided that the shape of the spectrum in coincidence with $\gamma$ rays only weakly depends on the $\gamma$ multiplicity. This fact is fulfilled for nuclei having low level density close to the ground state and rather narrow $(\simeq 2-3 \mathrm{MeV})$ TKEL distributions, as for the present near closed-shell nuclei.

By repeating this subtraction in steps of one degree over the entrance angular range $\left(\Delta \theta_{\text {lab }}=12^{\circ}\right)$ of PRISMA one obtains the elastic angular distribution whose ratio to Rutherford is shown in the bottom panel of Fig. 6, in comparison with the results of GRAZING calculations 22]. The very pronounced fall-off of the elastic cross section for large angles clearly indicates that the elastic scattering for this system is dominated by strong
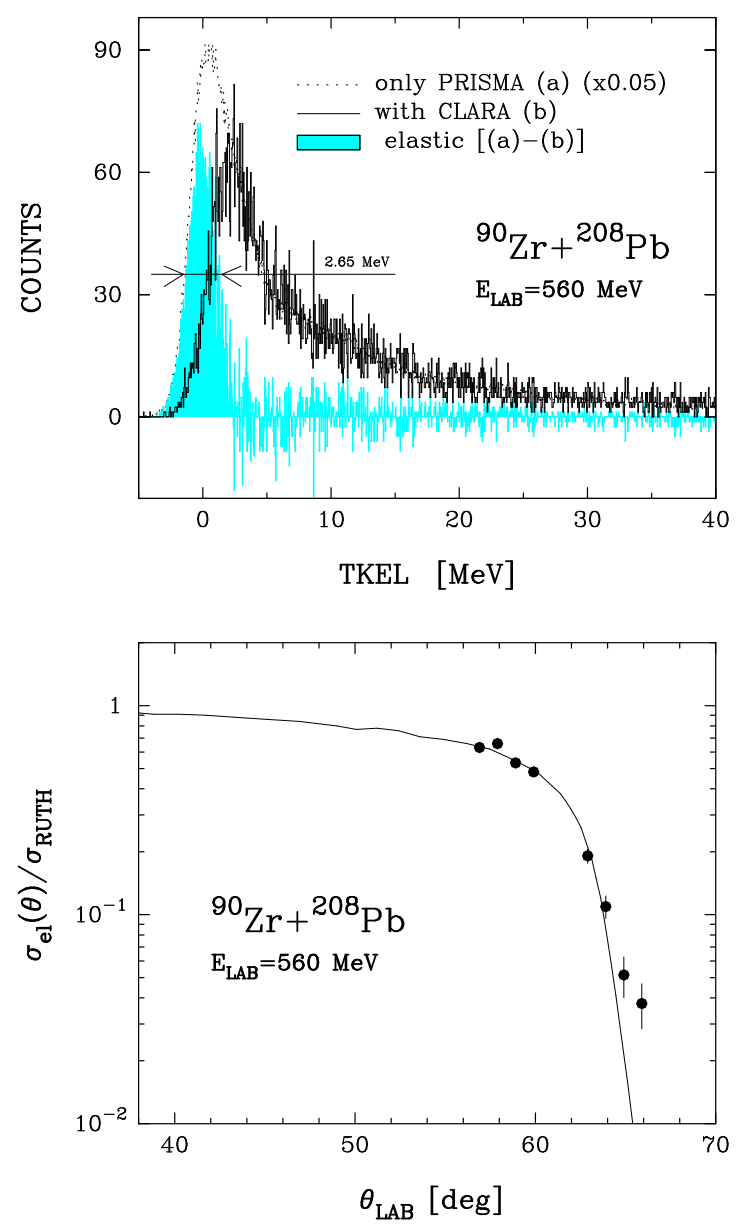

FIG. 6: (Color online) Top: Experimental angle integrated total kinetic energy loss distributions (TKEL) for ${ }^{90} \mathrm{Zr}$ in the ${ }^{90} \mathrm{Zr}+{ }^{208} \mathrm{~Pb}$ reaction (a) without coincidence with $\gamma$ rays and (b) with at least one $\gamma$ ray detected in CLARA. The two spectra are normalized in such a way that the high TKEL tails match. The gray area corresponds to the subtraction between the two spectra [(a)-(b)], giving a peak whose width is $\sim 2.65 \mathrm{MeV}$. Bottom: Experimental (points) and GRAZING calculated (curve) differential cross section for elastic scattering, normalized to Rutherford. Only statistical errors are included.

absorption. The good agreement between theory and experiment gives us confidence on the used potential and on the fact that the included reaction channels correctly describe the depopulation of the entrance channel (absorption).

Grazing reactions populate regions of excitation energy and spin differing substantially from the ones reached by fusion evaporation reactions. This can be here checked by a careful correlation of the TKEL spectra obtained with PRISMA with the coincident $\gamma$ rays of CLARA. The top panel of Fig. 7 shows the TKEL spectrum for ${ }^{92} \mathrm{Zr}$ from the ${ }^{90} \mathrm{Zr}+{ }^{208} \mathrm{~Pb}$ reaction, while the bottom panel shows the corresponding $\gamma$-ray spectra obtained without (top panel) and with (middle and bottom panels) dif- 

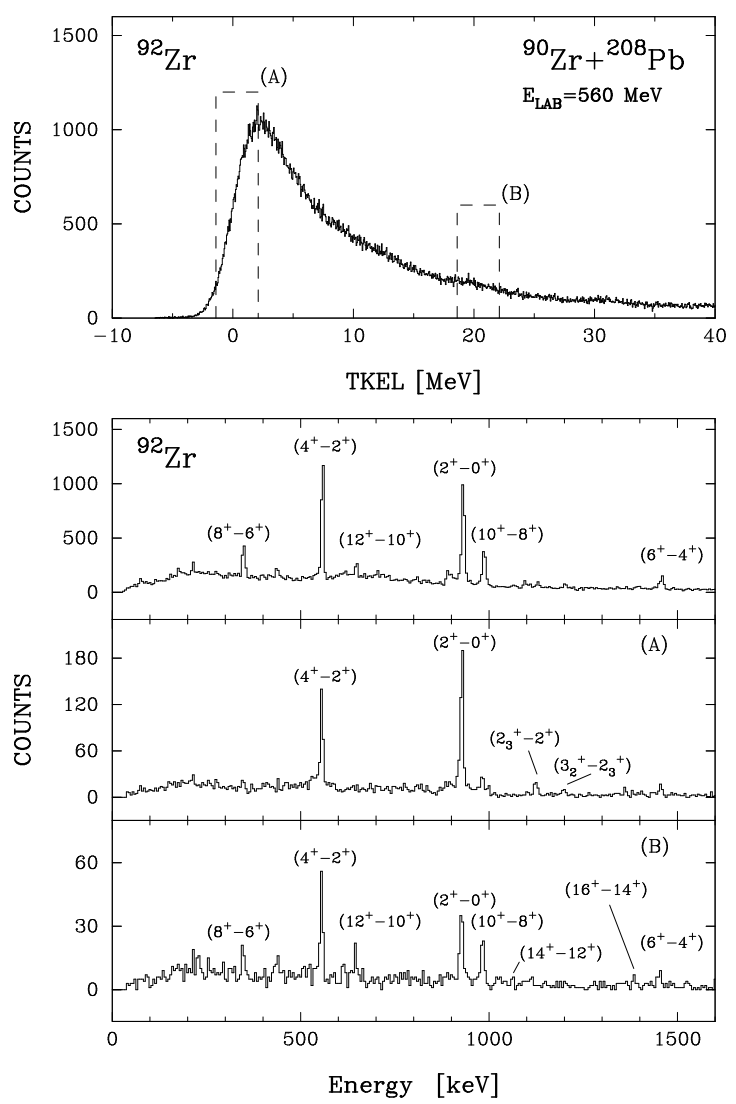

FIG. 7: Top: TKEL distribution for ${ }^{92} \mathrm{Zr}$ produced in the ${ }^{90} \mathrm{Zr}+{ }^{208} \mathrm{~Pb}$ reaction. Bottom: associated $\gamma$-ray spectra for ${ }^{92} \mathrm{Zr}$ without conditions on TKEL (top) and conditioned (middle and bottom) with different regions of TKEL distributions, marked as (A) and (B) in the TKEL spectrum and with gates $\simeq 3 \mathrm{MeV}$ wide.

ferent conditions on the TKEL. States up to spin $16^{+}$ and excitation energy of about $7.5 \mathrm{MeV}$, have been observed. By gating on the low TKEL region (A) the two lowest yrast transitions dominate the spectrum. By gating instead on the TKEL region around $20 \mathrm{MeV}$ (B) the spectrum displays transitions coming from the decay of high-spin states (we remark that the excitation energy of both light and heavy fragments is embedded into the TKEL distribution).

Exploiting the binary character of the studied reaction one can investigate the final mass partition influenced by evaporation processes. Gating with PRISMA on a specific $Z$ and $A$ (light partner) the velocity vector of the undetected heavy partner can be evaluated and applied for the Doppler correction of its corresponding $\gamma$ rays. In those spectra not only the $\gamma$ rays belonging to the primary binary partner are present but also the ones of the nuclei produced after evaporation takes place. For ${ }^{40} \mathrm{Ar}$ detected with PRISMA in the reaction ${ }^{40} \mathrm{Ca}+{ }^{96} \mathrm{Zr}$, we show in Fig. 8 the $\gamma$ spectra of the light and heavy partners. For this $-2 p+2 n$ channel, about $60 \%$ of the yield
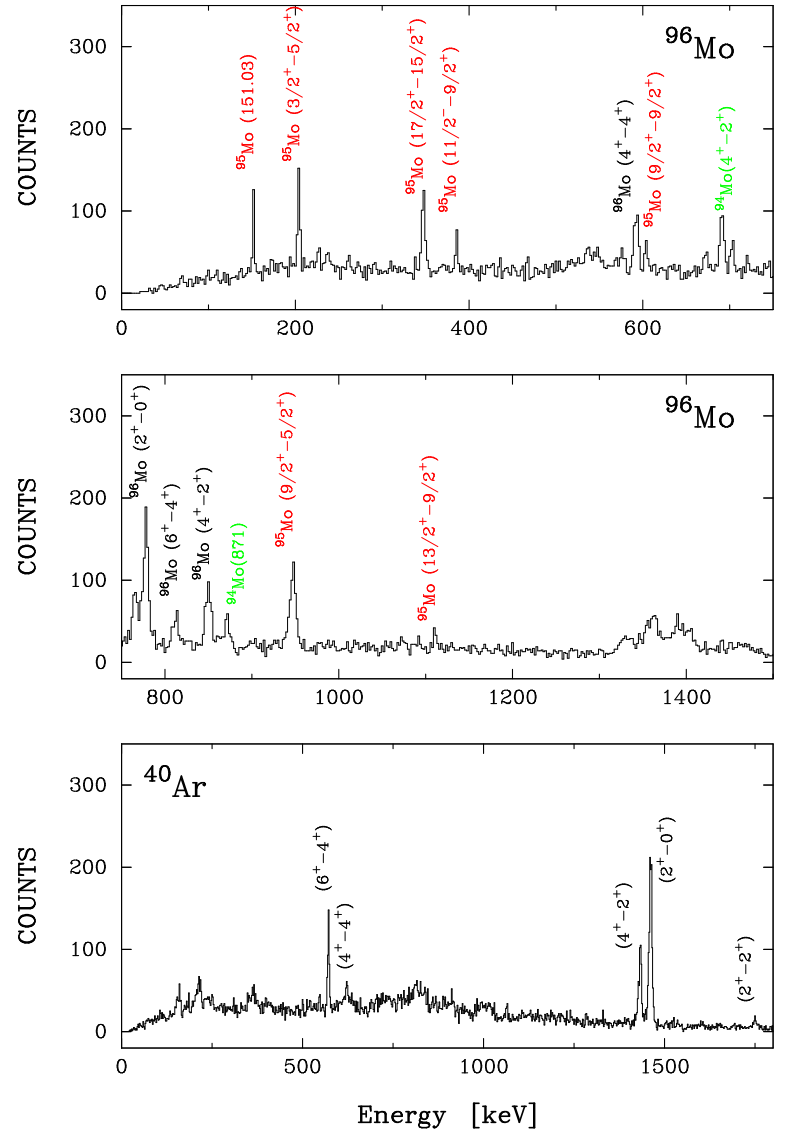

FIG. 8: (Color online) $\gamma$ spectra for the $-2 p+2 n$ channel in the reaction ${ }^{40} \mathrm{Ca}+{ }^{96} \mathrm{Zr}$ Doppler corrected for the heavy (top two frames) and light fragments (bottom frame). To have a better identification of the different $\gamma$ lines for the heavy fragment we used an expanded energy scale.

corresponds to ${ }^{96} \mathrm{Mo}$, while the rest is equally shared between isotopes corresponding to the evaporation of one and two neutrons, leading to ${ }^{95} \mathrm{Mo}$ and ${ }^{94} \mathrm{Mo}$, respectively. For even weaker channels like the $-4 p$ channel $\left({ }^{36} \mathrm{~S}\right)$ the $\gamma$ rays belonging to the primary binary partner $\left({ }^{100} \mathrm{Ru}\right)$ have negligible yield. On the other hand, for the $+2 n$ channel $\left({ }^{42} \mathrm{Ca}\right)$ more than $90 \%$ of the yield corresponds to the true binary partner, i.e. ${ }^{94} \mathrm{Zr}$. This behavior is closely connected with the observed TKEL. For the neutron pick-up channels the major contribution in the TKEL is close to the optimum $Q$ values $\left(Q_{\text {opt }} \simeq 0\right.$ ), while in the proton stripping channels larger TKEL are observed, thus the neutron evaporation has a stronger effect on the final mass partition. The importance of neutron evaporation in the modification of the final yield distribution was outlined in inclusive measurements [10, 11]. A direct signature of this effect was observed by correlating projectile-like and target-like fragment isotopic yields via $\gamma-\gamma$ coincidences [27, 28]. 


\section{ON THE STRUCTURE OF ${ }^{95}$ ZR AND ${ }^{42}$ CA}

The experimental yields have been interpreted with a model that explicitly treats the internal degrees of freedom of the two ions in terms of elementary modes, surface vibration and single particles. The excitation and transfer processes are mediated by the well known singleparticle form factors for the fermion degrees of freedom and by the collective form factors, nuclear plus coulomb, for the vibrational modes. Following this description heavy ion collisions provide a suitable tool for the studies of the particle-vibration coupling scheme, in fact it is through the excitation of these elementary modes that energy and angular momentum are transferred from the relative motion to the intrinsic degrees of freedom and that mass and charge are exchanged among the two parters of the collision. We remind that the de-excitation spectra of the produced isotopes may quite differ from the excitation ones. The quadrupole and octupole matrix elements generally play a major role in the excitation process, while the de-excitation may be dominated by very small components in the wave function.

The reactions we are analyzing in this manuscript are well suited for these studies since they involve magic and semi-magic nuclei. The spectra of the neighboring nuclei, populated in the reaction, comprise partly single particle or single hole states and partly states that involve combinations of single-particle or hole with a collective boson. Here we concentrate on spectra of ${ }^{95} \mathrm{Zr}$ populated via one-neutron pick-up reaction in the ${ }^{40} \mathrm{Ca}+{ }^{96} \mathrm{Zr}$ collision. Our analysis follows the one of Ref. [29] where the particle-phonon states in ${ }^{209} \mathrm{Bi}$ and ${ }^{207} \mathrm{~Pb}$, populated in deep-inelastic heavy ion collision, were analyzed.

The ${ }^{208} \mathrm{~Pb}$ nucleus constitutes the ideal laboratory for the study of particle-phonon states, being its first excited state the collective $3^{-}$at $2.62 \mathrm{MeV}$ and its first positive parity state the $2^{+}$at $4.05 \mathrm{MeV}$. The collectivity of the $3^{-}\left(B\left(E 3 ; 3^{-} \rightarrow 0^{+}\right)=34\right.$ W.u. $)$ is not dominated by few particle-hole components but derives from the cooperative action of many configurations. The ${ }^{96} \mathrm{Zr}$ nucleus presents a more complicated situation, its lowenergy spectra is dominated by a $2^{+}$state at $1.75 \mathrm{MeV}$ and by the $3^{-}$at $1.90 \mathrm{MeV}$. The last state is very collective $\left(B\left(E 3 ; 3^{-} \rightarrow 0^{+}\right)=51\right.$ W.u. $)$ and decays via an E1 transition to the $2^{+}$and via an E3 transition to the ground state [30]. The $\gamma$ spectra for ${ }^{96} \mathrm{Zr}$ and ${ }^{95} \mathrm{Zr}$ are shown in Fig. 91 they have been obtained by applying Doppler correction for target-like partners corresponding to ${ }^{40} \mathrm{Ca}$ and ${ }^{41} \mathrm{Ca}$ detected in PRISMA, respectively.

The ground state of ${ }^{95} \mathrm{Zr}$ is well described by a neutronhole in the $d_{5 / 2}$ orbital. By coupling this hole state with the $3^{-}$one expects a sextuplet of states $\left(1 / 2^{-}, 3 / 2^{-}\right.$, $\left.\ldots, 11 / 2^{-}\right)$at an energy close to the one of the $3^{-}$. Similarly, by coupling the same hole state to the first $2^{+}$one expects a quintuplet $\left(1 / 2^{+}, 3 / 2^{+} \ldots, 9 / 2^{+}\right)$at an energy close to the one of the $2^{+}$state in ${ }^{96} \mathrm{Zr}$. The reaction mechanism does not populate the components of the two multiplets uniformly but favors the stretched configura-
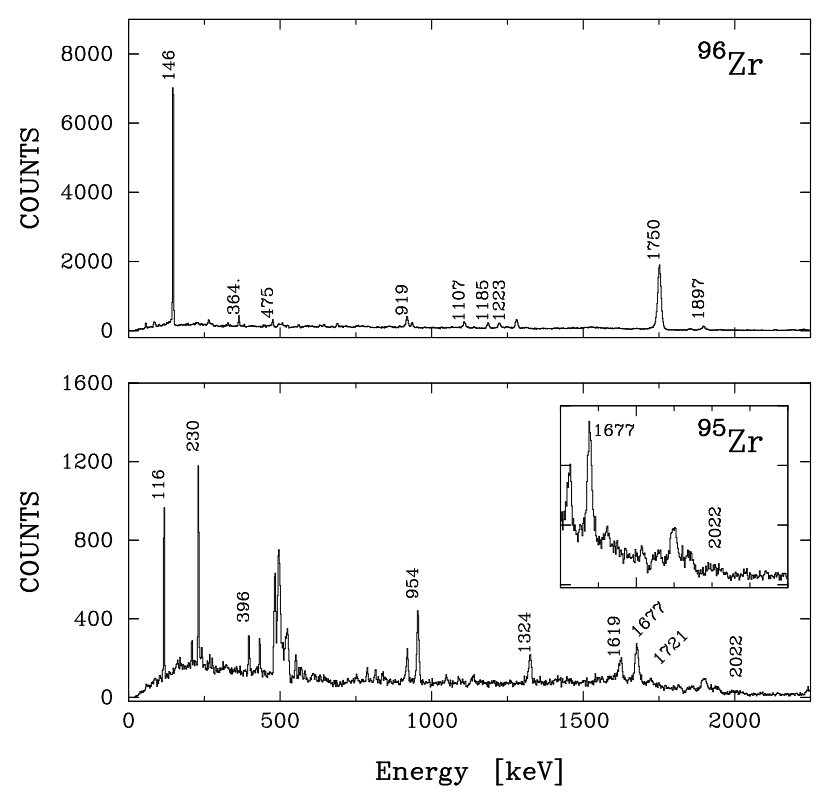

FIG. 9: Gamma-ray spectra for ${ }^{96} \mathrm{Zr}$ (top) and ${ }^{95} \mathrm{Zr}$ (bottom) obtained in the ${ }^{40} \mathrm{Ca}+{ }^{96} \mathrm{Zr}$ reaction (see text).

tions $11 / 2^{-}$and $9 / 2^{+}$since the transfer probability has its maximum at the largest angular momentum transfer. From the adopted levels of ${ }^{95} \mathrm{Zr}$, the state at $2025 \mathrm{keV}$ $(2025 \pm 7 \mathrm{keV})$ populated in $(p, d)$ and $\left({ }^{3} \mathrm{He}, \alpha\right)$ reactions (recognized as $9 / 2^{-}, 11 / 2^{-}$) 31] is a natural candidate for the stretched configuration $\mid 3^{-},\left(d_{5 / 2}\right)^{-1}>$. Very recently, the level scheme of ${ }^{95} \mathrm{Zr}$ has been re-measured 32 in heavy-ion induced fission reactions and the sequence of $\gamma$ rays of $229.7\left[11 / 2^{-} \rightarrow\left(9 / 2^{+}\right)\right], 115.8\left[\left(9 / 2^{+}\right) \rightarrow\right.$ $\left.\left(7 / 2^{+}\right)\right]$and $1676.8\left[\left(7 / 2^{+}\right) \rightarrow 5 / 2_{\text {g.s. }}^{+}\right] \mathrm{keV}$ has been proposed for the decay of the $11 / 2^{-}$state. In Fig. 9 the measured spectrum of ${ }^{95} \mathrm{Zr}$ shows very clearly all these transitions. In addition, a new transition at $\mathrm{E}_{\gamma}=2022 \mathrm{keV}$ is visible (the width of the $2022 \mathrm{keV}$ peak is partially affected by the wrongly Doppler corrected $3 / 2^{+} \rightarrow 7 / 2_{\text {g.s. }}^{-}$. transition from ${ }^{41} \mathrm{Ca}$ ), that we naturally interpret as the E3 decay of the $11 / 2^{-}$to the ground state. The intensity of this transition, relative to the E1, is very similar to the one observed in ${ }^{96} \mathrm{Zr}$ thus reinforcing our interpretation that the $11 / 2^{-}$is a member of a boson-hole $\mid 3^{-},\left(d_{5 / 2}\right)^{-1}>$ multiplet.

The closed-shell systems studied in this work are well suited for the identification of states reached via the addition and/or the removal of pairs of nucleons. Those states have been studied with light ion reactions and formed the basis for the identification of pairing vibration degrees of freedom in the nuclear medium 1]. In a previous experiment with ${ }^{40} \mathrm{Ca}+{ }^{208} \mathrm{~Pb}$ (cfr. [11] and Refs. therein) we have noticed that in order to obtain a good description of the experimental total cross sections for the different isotopes populated in the reaction one has to include, in the theoretical model, the degrees of freedom related to the 


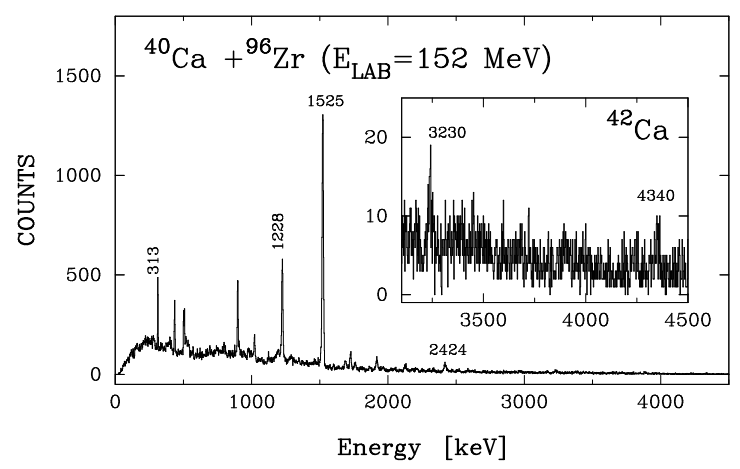

FIG. 10: $\gamma$-ray spectrum with expanded region in inlet for ${ }^{42} \mathrm{Ca}$ obtained in the ${ }^{40} \mathrm{Ca}+{ }^{96} \mathrm{Zr}$ reaction.

transfer of pairs of nucleons, both protons and neutrons. These degrees of freedom are treated as pair-vibrational modes and for their excitation we have used the form factors as provided by the macroscopic model. The influence of these degrees of freedom is particularly visible in the proton sector since the cross sections of proton stripping channels are much smaller than the neutron pick-up ones.

To have evidence of the excitation of these modes in the neutron sector we have analyzed in detail [33] the total kinetic energy loss (TKEL) spectra of ${ }^{42} \mathrm{Ca}$, the two neutron pick-up channel. Here most of the cross section is concentrated in a pronounced peak at an energy that is compatible with the excitation of a group of $0^{+}$states at $\sim 6 \mathrm{MeV}$ where a pairing-vibrational state should be located [1].

The present set-up should allow the observation of the decay pattern of the populated $0^{+}$states. In Fig. 10 we show the $\gamma$-spectrum for ${ }^{42} \mathrm{Ca}$ obtained in the reaction ${ }^{40} \mathrm{Ca}+{ }^{96} \mathrm{Zr}$. We observe here (see expanded region) a $\gamma$-transition at $4340 \mathrm{keV}$ which is consistent with a decay from a level at $5.8 \mathrm{MeV}$ to the $2_{1}^{+}$state [34, 35]. The limited statistics accumulated for this transition (we remark that such high energy $\gamma$ rays have a low photopeak efficiency) does not allow to deduce the spin of the populated level, though the distribution over the rings of CLARA shows an isotropic pattern but with very large error-bars. In the expanded $\gamma$ spectrum we also observe a $\gamma$ transition of $3230 \mathrm{keV}$, which is the main branch of the decay from the $2^{+}$state at $4760 \mathrm{keV}$, strongly populated in $(t, p)$ reactions [34, 35].

\section{SUMMARY}

Multi-nucleon transfer reactions have been studied with the large solid angle magnetic spectrometer PRISMA, where ions identification is achieved by reconstructing event-by-event the trajectory of the ions in the magnetic elements. Experimental yields have been compared with semi-classical models that include surface vibrations and single particle transfer modes and taking into account the effect of neutron evaporation. By coupling the PRISMA spectrometer with the high resolution CLARA $\gamma$-array we showed how the transfer process populates reaction products in regions of angular momentum and excitation energy quite different from fusionevaporation processes. This has been exploited to gain information on states that can be interpreted along the particle-vibration coupling scheme. In particular, we discussed the low energy spectrum of ${ }^{95} \mathrm{Zr}$ populated in oneneutron pick-up reactions. Very preliminary results on the possible pairing vibration in ${ }^{42} \mathrm{Ca}$ are also reported.

\section{Acknowledgments}

The authors are grateful to the LNL Tandem-ALPI stuff for providing us with the good quality beams and the target laboratory for the excellent target preparation. This work was partly supported by the European Community FP6 - Structuring the ERA - Integrated Infrastructure Initiative - contract EURONS No. RII3-CT2004-506065. This work was, also, supported in part by the Croatian Ministry of Science, Education and Sports, Grant No. 0098-1191005-2890.
[1] R.A. Broglia, O. Hansen and C. Riedel, Advances in Nuclear Physics, edited by M. Baranger and E. Vogt, Plenum, New York, 1973, Vol. 6, p.287.

[2] R.A. Broglia and A. Winther, Heavy Ion Reactions (Addison-Wesley Pub. Co., Redwood City CA, 1991).

[3] C.Y. Wu, W. von Oertzen, D. Cline and M. Guidry, Annu. Rev. Nucl. Part. Sci. 40, 285 (1990).

[4] J. Schirmer, D. Habs, R. Kroth, N. Kwong, D. Schwalm, M. Zirnbauer, and C. Broude, Phys. Rev. Lett. 53, 1897 (1984).

[5] H.J. Wollersheim, P.Egelhof, H. Emling, J. Gerl, W. Henning, R. Holzmann, R. Schmidt, R.S. Simon, N. Martin, G. Eckert, Th.W. Elze, K. Stelzer, R. Kulessa, G. Duchene, B. Haas, J.C. Merdinger, J.P.Vivien, J.de Boer, E.
Hauber, K. Kaiser, P.von Brentano, R. Reinhardt, R. Wirowski, R. Julin, C. Fahlander, I. Thorslund, and H. Kluge, Z. Phys. A341, 137 (1992).

[6] K. Vetter, A.O. Macchiavelli, D. Cline, H. Amro, S.J. Asztalos, B.C. Busse, R.M. Clark, M.A. Deleplanque, R.M. Diamond, P. Fallon, R. Gray, R.V.F. Janssens, R. Krücken, I.Y. Lee, R.W. Macleod, E.F. Moore, G.J. Schmidt, M.W. Simon, F.S. Stephens, and C.Y. Wu, Phys. Rev. C 58, R2631 (1998).

[7] M. Rejmund, K.H. Maier, R. Broda, B. Fornal, M. Lach, J. Wrzesinski, J. Blomqvist, A. Gadea, J. Gerl, M. Gorska, H. Grawe, M. Kaspar, H. Schaffner, Ch. Schlegel, R. Schubart, and H.J. Wollersheim, Eur. Phys. J. A8, 161 (2000) and Refs. therein. 
[8] A. Winther, Nucl. Phys. A572, 191 (1994); Nucl. Phys A594, 203 (1995).

[9] E. Vigezzi and A. Winther, Ann. Phys. (N.Y.) 192, 432 (1989).

[10] L. Corradi, A.M. Stefanini, C.J. Lin, S. Beghini, G. Montagnoli, F. Scarlassara, G. Pollarolo, and A. Winther, Phys. Rev. C 59, 261 (1999).

[11] S. Szilner, L. Corradi, G. Pollarolo, S. Beghini, B.R. Behera, E. Fioretto, A. Gadea, F. Haas, A. Latina, G. Montagnoli, F. Scarlassara, A.M. Stefanini, M. Trotta, A.M. Vinodkumar, and Y. Wu, Phys. Rev. C 71 (2005) 044610.

[12] L. Corradi, G. de Angelis, A. Gadea, G. Maron, D.R. Napoli, A.M. Stefanini, S. Beghini, D. Bazzacco, G. Montagnoli, P. Pavan, F. Scarlassara, C.A. Ur, J.H. He, C. Fahlander, G. Pollarolo, and F. Cerutti, Phys. Rev. C 61 (2000) 024609.

[13] A.M. Stefanini, L. Corradi, G. Maron, A. Pisent, M. Trotta, A.M. Vinodkumar, S. Beghini, G. Montagnoli, F. Scarlassara, G.F. Segato, A. De Rosa, G. Inglima, D. Pierroutsakou, M. Romoli, M. Sandoli, G. Pollarolo, and A. Latina, Nucl. Phys. A701, 217c (2002).

[14] H.Savajols and VAMOS collaboration, Nucl. Phys. A654 (1999) 1027c; http://www.ganil.fr/vamos

[15] A. Gadea, D.R. Napoli, G. de Angelis, R. Menegazzo, A.M. Stefanini, L. Corradi, M. Axiotis, L. Berti, E. Fioretto, T. Kroell, A. Latina, N. Marginean, G. Maron, T. Martinez, D. Rosso, C. Rusu, N. Toniolo, S. Szilner, M. Trotta, D. Bazzacco, S. Beghini, M. Bellato, F. Brandolini, E. Farnea, R. Isocrate, S.M. Lenzi, S. Lunardi, G. Montagnoli, P. Pavan, C.R. Alvarez, F. Scarlassara, C.A. Ur, N. Blasi, A. Bracco, F. Camera, S. Leoni, B. Million, M. Pignanelli, G. Pollarolo, A. DeRosa, C. Inglima, M. La Commara, G. La Rana, D. Pierroutsakou, M. Romoli, M. Sandoli, P.G. Bizzeti, A.M. Bizzeti-Sona, G. Lo Bianco, C.M. Petrache, A. Zucchiatti, P. Cocconi, B. Quintana, C. Beck, D. Curien, G. Duchene, F. Haas, P. Medina, P. Papka, J. Durell, S.J. Freeman, A. Smith, B. Varley, K. Fayz, V. Pucknell, J. Simpson, W. Gelletly, and P. Regan, Eur. Phys. J. A 20193 (2004).

[16] S.L. Shepherd, P.J. Nolan, D.M. Cullen, D.E. Appelbe, J. Simpson, J. Gerl, M. Kaspar, A. Kleinboehl, I. Peter, M. Rejmund, H. Schaffner, C. Schelgel, and G. de France, Nucl. Instr. and Methods in Phys. Res. A 434, 373 (1999).

[17] Fusion06: Int. Conf. on Reaction Mechanisms and Nuclear Structure at the Coulomb barrier, S.Servolo (Venezia), Italy,19-23 March 2006, AIP Proceedings Series, Vol. 853, Melville (New York), L. Corradi, D. Ackermann, E. Fioretto, A. Gadea, F. Haas, G. Pollarolo, F. Scaralassara, S. Szilner, and M. Trotta eds., p. 29 and p.43.

[18] G. Montagnoli, A.M. Stefanini, M. Trotta, S. Beghini, M. Bettini, F. Scarlassara, V. Schiavon, L. Cor- radi, B.R. Behera, R. Fioretto, A. Gadea, A. Latina, S. Szilner, L. Donà, M. Rigato, N.A. Kondratiev, A. Yu. Chizhov, G. Kniajeva, E.M. Kozulin, I.V. Pokrovskiy, V.M. Voshressensky, and D. Ackermann, Nucl. Instr. and Meth. in Phys. Res. A 547, 455 (2005).

[19] S. Beghini, L. Corradi, E. Fioretto, A. Gadea, A. Latina, G. Montagnoli, F. Scarlassara, A.M. Stefanini, S. Szilner, M. Trotta, and A.M. Vinodkumar, Nucl. Instr. and Meth. in Phys. Res. A 551364 (2005).

[20] A. Latina, Ph.D. Thesis, Torino University 2004, unpublished.

[21] R. Shima, T. Ishihara, and T. Mikumo, Nucl. Instrum. Meth. 200, 605 (1982).

[22] A. Winther, program GRAZING, htpp:/www.to.infn.it/ nanni/grazing.

[23] K.E. Rehm, Annu. Rev. Nucl. Part. Sci. 41, 429 (1991).

[24] C.H. Dasso, G. Pollarolo and A. Winther, Phys. Rev. Lett. 73, 1907 (1994).

[25] R.A. Broglia, G. Pollarolo, and A. Winther, Nucl. Phys. A 361, 307 (1981).

[26] G. Pollarolo, R.A. Broglia, and A. Winther, Nucl. Phys. A 406, 369 (1983).

[27] S.J. Asztalos, I.Y. Lee, K. Vetter, B. Cederwall, R.M. Clark, M.A. Deleplanque, R.M. Diamond, P. Fallon, K. Jing, L. Phair, A.O. Macchiavelli, F.S. Stephens, G.J. Wozniak, L.A. Bernstein, D.P. McNabb, P.F. Hua, D.G. Sarantites, J.X. Saladin, C.-H. Yu, and J.A. Cizewski, Phys. Rev. C 61, 014602 (1999).

[28] R. Broda, J. of Phys. G 32, R151 (2006).

[29] M. Kadi, P.E. Garrett, M. Yeh, S.W. Yates, T. Belgya, A.M. Oros-Peusquens and K. Heyde, Phys. Rev. C 61, 034307 (2000).

[30] M.R. Bhat, Nuclear Data Sheets 82, 547 (1997).

[31] C.R. Bingham and G.T. Fabian, Phys. Rev. C 7, 1509 (1973)

[32] D. Pantelica, I.Gh. Stefan, N. Nica, M.-G. Porquet, G. Duchene, A. Astier, S. Courtin, I. Deloncle, F. Hoellinger, A. Bauchet, N. Buforn, L. Donadille, O. Dorvaux, J. Duprat, B.J.P. Gall, C. Gautherin, T. Kutsarova, S. Lalkovski, R. Lucas, M. Meyer, A. Minkova, A. Prevost, N. Redon, N. Schulz, H. Sergolle, O. Stezowski, Ts. Venkova, and A. Wilson, Phys. Rev. C 72, 024304 (2005).

[33] S. Szilner, L. Corradi, F. Haas, G. Pollarolo, S. Beghini, B.R. Behera, E. Caurier, E. Fioretto, A. Gadea, A. Latina, G. Montagnoli, F. Nowacki, F. Scarlassara, A.M. Stefanini, M. Trotta, A.M. Vinodkumar, and Y.W. Wu, Eur. Phys. J. A 21, 87 (2004).

[34] J.H. Bjerregaard, O. Hansen, O. Nathan, R. Chapman, S. Hinds and R. Middleton, Nucl. Phys. A 103 (1967) 33.

[35] D.C.Williams, J.D.Knight and T.Leland, Phys. Rev. 164, 1419 (1967). 